\title{
Google als kartellrechtliches Problem?
}

\author{
Andreas Heinemann *
}

Google ist im Besitz beherrschender Stellungen auf mehrseitigen Märkten und darf die Position der Stärke nicht missbrauchen. Ausschliesslichkeitsbindungen sind problematisch. Hingegen sind auch Marktbeherrscher in der Ausgestaltung ihrer Produkte frei; ein allgemeines Gebot der Suchneutralität existiert nicht. Google muss aber Marktverschliessungen durch Zugangsgewährung auflösen und unterliegt bei den natürlichen Trefferlisten einem Willkürverbot und Verfahrensanforderungen. Die kartellrechtlichen Vorgaben sollten darauf abzielen, die Innovationstätigkeit aller Akteure zu fördern und die Märkte offen zu halten.

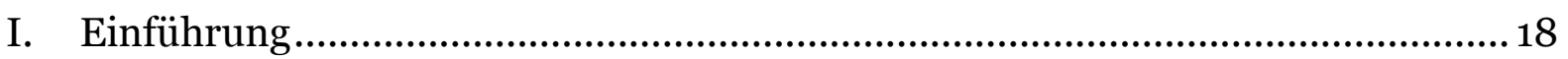

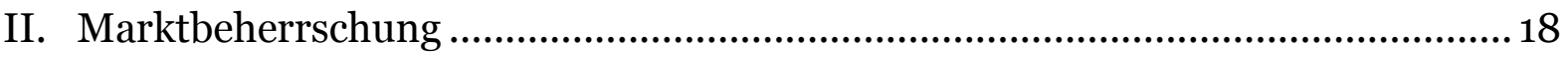

1. Funktionsweise von Suchmaschinen .......................................................... 18

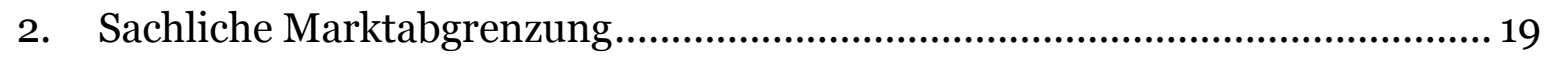

3. Marktbeherrschende Stellung ...............................................................23

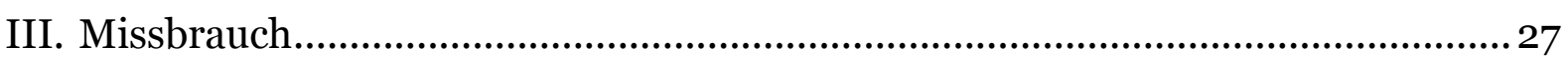

1. Privilegierung eigener Suchdienste......................................................27

2. Übernahme der Inhalte von Konkurrenten ...............................................28

3. Ausschliesslichkeitsbindungen bei Suchmaschinenwerbung .......................29

4. Übertragbarkeit von Werbekampagnen .................................................29

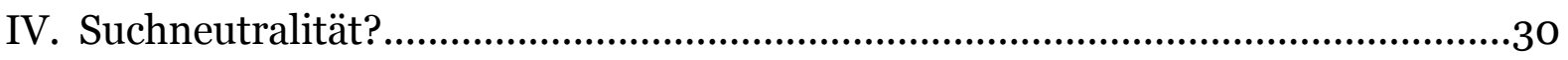

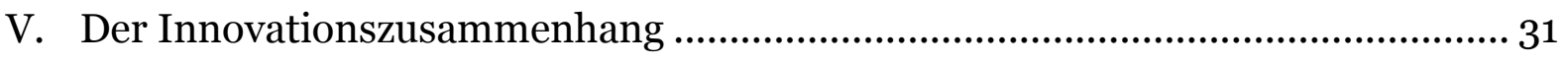

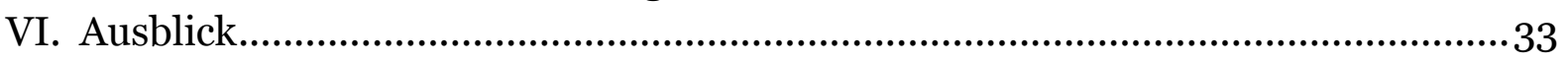

Zitiervorschlag: Andreas Heinemann, Google als kartellrechtliches Problem?, in: sui-generis 2015, S. 17

URL: $\quad$ sui-generis.ch/11

* Professor an der Universität Zürich, Vizepräsident der schweizerischen Wettbewerbskommission. Der Verfasser gibt seine persönliche Meinung wieder.

Der Text beruht auf dem Vortrag, den der Verfasser am 19. Januar 2015 am Zentrum für Europäisches Wirtschaftsrecht der Universität Bonn gehalten hat. Eine stark erweiterte Fassung des Beitrags erscheint in der Schriftenreihe des Zentrums, die über www.zew.uni-bonn.de/publikationen zugänglich ist. 


\section{Einführung}

1 Ende 2010 hat die Europäische Kommission ein Kartellverfahren gegen Google eingeleitet. Beschwerden von Wettbewerbern waren eingegangen, welche die Kommission im Lauf des Verfahrens auf vier Vorwürfe verdichtete. In den Jahren 2013 und 2014 wurde versucht, das Verfahren durch Verpflichtungszusagen nach Art. 9 der europäischen Kartellverordnung (VO 1/20031) abzuschliessen. Dies gelang aber nicht vor dem Amtsantritt der neuen Kommission am 1. November 2014.² Bei Abschluss dieses Manuskripts ist der Ausgang des Verfahrens offen.

Der vorliegende Beitrag befasst sich mit der Frage, ob die erhobenen Vorwürfe durch das Verbot des Missbrauchs marktbeherrschender Stellungen in Art. 102 AEUV / Art. 7 KG gedeckt sind. ${ }^{3}$ Es ist also zu klären, ob Google marktbeherrschend ist (II.), und ob das Unternehmen eine marktbeherrschende Stel-

1 Verordnung (EG) Nr. 1/2003 des Rates vom 16.12.2002 zur Durchführung der in den Artikeln 81und 82 des Vertrags niedergelegten Wettbewerbsregeln, ABl. 2003 L 1/1.

2 Im Gegensatz hierzu hat die US-amerikanische Federal Trade Commission ihr Google-Verfahren durch ein consent agreement beendet. Der gravierendste, das Kerngeschäft des Unternehmens betreffende Vorwurf, nämlich derjenige der wettbewerbswidrigen Manipulation des Suchalgorithmus, wurde fallengelassen, s. Federal Trade Commission, Pressemitteilung vom 3.1.2013 <www.ftc.gov/opa/2013/01/google.shtm>.

3 Aufgrund der thematischen Ausrichtung dieses Beitrags auf das Kartellverfahren der Europäischen Kommission stehen hier Fragen despublic enforcement im Vordergrund. Zu privatrechtlichen Folgen (aus der Perspektive des schweizerischen Rechts) s. Hürlimann, Suchmaschinenhaftung - Zivilrechtliche Verantwortlichkeit der Betreiber von InternetSuchmaschinen aus Urheber-, Marken-, Lauterkeits-, Kartell- und Persönlichkeitsrecht, 2012, S. 59 ff., 73 ff., 151 ff. lung missbraucht hat (III.).Nach einer Diskussion des Konzepts der Suchneutralität (IV.) und einer Betrachtung des Innovationszusammenhangs (V.) erfolgt ein allgemeiner Ausblick (VI.).

\section{Marktbeherrschung}

\section{Funktionsweise von Suchmaschi- nen}

\section{a) Suchvorgang}

3 Suchmaschinen machen die Inhalte des «weltweiten Netzes» (World Wide Web ${ }^{4}$ ) zugänglich und reduzieren hierdurch Komplexität.5 Die Roboter bzw. Crawler des Suchmaschinenanbieters durchforsten das Netz und führen dessen Inhalte in einem Index auf. Richtet ein Nutzer eine Anfrage an eine Suchmaschine, durchsucht diese den Index, nicht etwa das Netz, was die hohe Reaktionsgeschwindigkeit möglich macht. Der Algorithmus der Suchmaschine entscheidet über die Platzierung, also das Ranking von Webseiten auf der Trefferliste. Die Einträge auf dieser Liste werden auch als «natürliche» oder «organische» Ergebnisse bezeichnet, weil sie nicht gekauft sind, sondern sich aus der Anwendung sachlicher Kriterien ergeben. Der GoogleAlgorithmus verfügt über mehr als 200 Kriterien und verzeichnet mehr als 500 Änderungen pro Jahr. ${ }^{6}$ Das bekannteste Kriterium ist der PageRank-Algorithmus,

4 Das World Wide Web ist nur ein Teil bzw. eine Anwendung des Internet. Zur sprachlichen Vereinfachung werden in diesem Artikel die Begriffe Internet und Netz synonym verwendet.

5 S. Heinemann, Reduktion von Komplexität durch Suchmaschinen: Ökonomische Grundlagen und kartellrechtliche Vorgaben, in Brandi-Dohrn/Lejeune (Hrsg.), Recht 2.0 - Informationsrecht zwischen virtueller und realer Welt, 2008, S. $13 \mathrm{ff}$.

6 S. die eigenen Angaben des Unternehmens unter <www.google.com/intl/de/insidesearch/ howsearchworks/algorithms.html>. 
der die Linkpopularität einer Webseite bewertet, z.B. die Anzahl der Links von anderen Webseiten sowie deren Qualität und Relevanz.

4 Google bietet nicht nur Suche nach Textdateien an, sondern beispielsweise auch nach Bildern und Videos. Dutzende anderer Dienste stehen zur Verfügung, wie z.B. Landkarten, Übersetzung und Büchersuche. Diese Dienste machen Google noch attraktiver, generieren mehr «traffic» und steigern hierdurch die Werbeeinnahmen. Die jüngere Entwicklung geht hierüber noch hinaus: Suchmaschinen streben danach, die Antwort auf die gestellte Frage direkt zu geben, anstatt lediglich auf andere Seiten zu verweisen.7 Hieraus entsteht ein Interessenkonflikt: Anstatt eine Suchanfrage durch den Verweis auf die einschlägigste Seite eines Drittanbieters zu beantworten, liegt es nahe, eigene Dienste zu bevorzugen. ${ }^{8}$

\section{b) Horizontale und vertikale Suchmaschinen}

5 Unterschieden wird zwischen horizontalen und vertikalen Suchmaschinen. Horizontale Suchmaschinen sind allgemeiner Natur und liefern Treffer für beliebige Anfragen. Demgegenüber sind vertikale Suchmaschinen auf besondere Themen spezialisiert, z.B. auf Hotels, Restaurants, Reisen, lokale Suche, Shopping oder Preisvergleiche. Im GoogleKartellverfahren lautet eine wichtige Frage, inwieweit Marktmacht einer horizontalen Suchmaschine eingesetzt wer-

\footnotetext{
S. hierzu Crane, Search Neutrality and Referral Dominance, 8 Journal of Competition Law \& Economics 459 (2012).

8 Crane, After Search Neutrality: Drawing a Line Between Promotion and Demotion, 9 I/S: A Journal of Law and Policy for the Information Society 397, 400 (2014).
}

den darf, um die eigenen vertikalen Dienste zu fördern.

\section{c) Geschäftsmodell}

6 Die Nutzer der Suchmaschine zahlen nicht für die in Anspruch genommene Dienstleistung. Die Betreiber der Suchmaschine verdienen ihr Geld mit Werbung. In seinem AdWords-Programm versteigert Google Werbeplätze auf den eigenen Ergebnisseiten. Die Werbung erscheint typischerweise oberhalb der natürlichen Trefferliste oder rechts daneben. Über AdSense vermittelt Google Werbung an die Webseiten Dritter und erhält für die Vermittlungsdienstleistung einen Teil des bezahlten Entgelts. Typisch für Suchmaschinenwerbung ist das Prinzip pay per click: Nicht das Aufschalten der Werbung führt zu Zahlungspflichten, sondern erst das Klicken auf die Werbung durch einen Nutzer.

\section{Sachliche Marktabgrenzung}

\section{a) Vorbemerkung}

7 Für die Abgrenzung der relevanten Märkte ist zwischen der Nutzung der Suchmaschine und dem Verkauf und der Vermittlung von Werbung zu unterscheiden. Da die Nutzer die Suchmaschine nicht (jedenfalls nicht primär) deshalb konsultieren, um zu Werbung zu gelangen, ist auch das Verhältnis zwischen Suchmaschine und Inhalteanbietern von Bedeutung. Entsprechend kommen drei relevante Märkte in Betracht, nämlich der Nutzer- oder Suchmarkt, der Werbemarkt (bzw. die Werbemärkte) sowie der Markt für die Aufnahme von Webseiten in den Index einer Suchmaschine. 9

$9 \overline{\text { S. z.B. Heinemann (oben Fn. 5), S. } 18 \text { ff.; Höppner, }}$ Das Verhältnis von Suchmaschinen zu Inhaltean- 
Diese Märkte stehen allerdings nicht unverbunden nebeneinander, so dass zunächst auf das Phänomen der zweiseitigen Märkte bzw. mehrseitigen Plattformen einzugehen ist.

\section{b) Zweiseitige Märkte/Mehrseitige Plattformen}

8 Zweiseitige Märkte (oder allgemeiner: mehrseitige Plattformen) bringen mindestens zwei Nutzergruppen zusammen, die aufeinander in Form einer Externalität einwirken. ${ }^{10}$ Aufgrund dieser Interaktion können die Betreiber ihre Plattform nicht lediglich auf die Interessen einer Nutzergruppe ausrichten, sondern müssen alle beteiligten Nutzergruppen im Auge behalten. Das Ziel der Gewinnmaximierung besteht nicht isoliert in Bezug auf einzelne Nutzergruppen, sondern auf die Plattform insgesamt.

9 Im Internet existieren zahlreiche mehrseitige Plattformen, z.B. soziale Netzwerke und Suchmaschinen. Je mehr Nutzer die Plattform hat, desto attraktiver ist sie für die werbende Wirtschaft. Die maximale Verbreitung der Plattform wird damit wichtiger als die Generierung von Einnahmen gegenüber jeder einzelnen Nutzergruppe. Die Preise gegenüber einer Nutzergruppe können also sehr niedrig sein, weil die grösstmögliche Verbreitung der Plattform zu hohen Einnahmen bei der anderen Nutzergruppe führt. Im Extremfall sinkt der Preis gegenüber der einen Nutzergruppe (jedenfalls pekuniär)

bietern an der Schnittstelle von Urheber- und Kartellrecht, WRP 2012, 625

(626); Monopolkommission, Zwanzigstes Hauptgutachten 2012/2013 - Eine Wettbewerbsordnung für die Finanzmärkte, 2014, Nr. 29.

10 Grundlegend Rochet/Tirole, Platform Competition in Two-Sided Markets, 1 Journal of the European Economic Association 990 (2003). auf null. ${ }^{11}$ So verhält es sich beispielsweise bei den Suchmaschinen, für deren Leistungen der Nutzer keine Geldzahlung erbringt.

\section{c) Nutzermarkt}

1o Das Konzept der mehrseitigen Plattformen ist von besonderer Bedeutung für die Definition des relevanten Nutzermarktes. Im Zentrum eines schon lange geführten Meinungsstreits steht die Frage, ob ein Markt im kartellrechtlichen Sinn Entgeltlichkeit voraussetzt. Die Befürworter dieses Kriteriums machen geltend, dass einem Markt zwingend ein entgeltliches Austauschverhältnis zugrunde liegen müsse. ${ }^{12}$ Diesem Standpunkt ist zuzugestehen, dass ein Markt der Ort ist, an dem Angebot und Nachfrage aufeinandertreffen, also ein Austausch stattfindet. Im Kartellrecht gilt aber eine wirtschaftliche Betrachtungsweise: Beim Zusammenspiel von Angebot und Nachfrage muss es nicht unbedingt zu einem Vertragsschluss im juristischen Sinn kommen. Auch muss die Gegenleistung der Nachfrager nicht unbedingt pekuniärer Natur sein. Entscheidend ist, dass der Anbieter einen wirtschaftlichen Gegenwert für seine Leis-

$11 \overline{\text { Dewenter/Rösch, Einführung in die neue Ökonomie }}$ der Medienmärkte, 2014, S. 123 f. Zu den kartellrechtlichen Problemen von Gratisprodukten s. Gal/Rubinfeld, The Hidden Costs of Free Goods: Implications for Antitrust Enforcement, NYU School of Law, Law \& Economics Research Paper Series, Working Paper No. 14-44, 2015 $<$ http://papers.ssrn.com/sol3/papers.cfm?abstract_id $=2529425>$.

12 S. z.B. Kersting/Dworschak, Leistungsschutzrecht für Presseverlage: Müsste Google wirklich zahlen? Eine kartellrechtliche Analyse, NZKart 2013, 46 (47 f.); Wiedemann, in Wiedemann (Hrsg.), Handbuch des Kartellrechts, 2. Aufl. 2008, § 23 Rn. 7; differenzierend Schulz/Held/Laudien, Suchmaschinen als Gatekeeper in der öffentlichen Kommunikation, 2005, S. 84. 
tung bekommt. ${ }^{13}$

11

Im Zusammenhang mit Suchmaschinen erbringt der Nutzer seine Gegenleistung für den Suchdienst auf zweierlei Weise: Einerseits schenkt er der Suchmaschine und insbesondere deren Trefferliste Aufmerksamkeit, ${ }^{14}$ wodurch er interessant für die werbende Wirtschaft wird, die Präsenz um die Trefferliste herum markiert. Und andererseits erhält der Betreiber der Suchmaschine Einsicht in das Nutzerverhalten, was ihm die Verbesserung der Suchmaschine erlaubt. Der Nutzer zahlt also mit seinen eigenen Daten, getreu dem Motto «If you're not paying for the product, you are the product». Bereits der Wettbewerb um die Aufmerksamkeit sollte ausreichen, eigene Nutzermärkte im Sinne des Kartellrechts anzunehmen. Das Konzept der mehrseitigen Plattformen hat gezeigt, dass die Frage des Entgelts eine Zufälligkeit darstellt, die von den konkreten Eigenschaften der jeweiligen Plattform abhängt. Die kontinuierlichen Innovationen durch Google veranschaulichen, welch hohen Wert das Unternehmen auf die Aufmerksamkeit der Nutzer legt, und wie intensiv der Wettbewerb um deren Aufmerksamkeit geführt wird. Aus diesem Grund sind eigene Nutzermärkte anzunehmen, und zwar schon unabhängig von dem lediglich unterstützenden Argument, dass die Nutzer auch mit ihren Daten zahlen. 15

13 Weber/Volz, Kartellrechtlicher Handlungsbedarf im Lichte potenzieller Meinungsmacht von Suchmaschinen, WuW 2015, unter II.2 (im Erscheinen).

14 S. grundlegend Franck, Ökonomie der Aufmerksamkeit - Ein Entwurf, 1998.

15 S. auch Körber, Google im Fokus des Kartellrechts, WRP 2012, 761 (764), der zu Recht Konsistenz mit den Fällen einfordert, in denen die europäischen Instanzen eigene Märkte für (unentgeltlich verteilte)
12 Da allgemeine Suchdienste nur in Ausnahmefällen durch besondere Suchdienste substituierbar sind, nämlich wenn nach einer Information gesucht wird, die zufälligerweise in den Spezialisierungsbereich einer vertikalen Suchmaschine fällt, ist von getrennten Nutzermärkten für horizontale und vertikale Suche auszugehen. ${ }^{16}$

\section{d) Werbemärkte}

13 Unternehmen sind ständig auf der Suche nach dem richtigen Werbemix. Geworben werden kann auf traditionellen Wegen, beispielsweise in Zeitungen und Zeitschriften, in Radio und Fernsehen, auf Plakaten oder bei Veranstaltungen. Hinzugetreten ist die Werbung im Internet in ihren verschiedenen Formen, z.B. Display-Werbung in Form von Werbebannern oder Suchmaschinenwerbung, indem die jeweils passende Werbung zu den Suchanfragen der Nutzer geschaltet wird. Werbeaktivitäten online und offline werden als getrennte Märkte wahrgenommen, da erstere gezielter eingesetzt werden können. ${ }^{17}$

14 Ungeklärt ist hingegen, ob innerhalb der Onlinewerbung eine noch feinere Markt-

Browser, Media Player und Konsumentenkommunikationsdienste annahmen.

16 In diesem Sinn auch Dewenter/Rösch/Terschüren, Abgrenzung zweiseitiger Märkte am Beispiel von Internetsuchmaschinen, NZKart 2014, 387 (392); van Loon, The Power of Google: First Mover Advantage or Abuse of a Dominant Position?, in: Lopez-Tarruella (Hrsg.), Google and the Law Empirical Approaches to Legal Aspects of Knowledge-Economy Business Models, 2012, S. 25; Monopolkommission (oben Fn. 9),

Nr. 29; Weber, Competition Law Issues in the Online World, 2013 <ssrn.com/link/20th-St-GallenICF.html>, S. 9.

17 In diesem Sinn Europäische Kommission, 3.10.2014, COMP/M.7217 - Facebook/WhatsApp, Nr. 75. 
abgrenzung vorzunehmen ist, ob beispielsweise ein eigener relevanter Markt für suchgebundene Werbung ( «Suchmaschinenwerbung») anzunehmen ist. ${ }^{18}$ Für diesen Standpunkt spricht die Tatsache, dass Suchmaschinenwerbung Interessenten am präzisesten erreicht. ${ }^{19}$ Fragen der Marktabgrenzung sind allerdings empirischer Natur. Obwohl viel für die Annahme eines eigenen Marktes für Suchmaschinenwerbung spricht, ist die Frage nach der richtigen Marktabgrenzung der (Online-)Werbemärkte derzeit als offen zu bezeichnen. ${ }^{20}$

\section{e) Aufnahme in den Index}

Unternehmen haben ein starkes Interesse daran, in den Index eines Suchmaschinenbetreibers zu gelangen, da sie nur auf diesem Weg über Suchanfragen in die Trefferliste gelangen können. Es gilt der Grundsatz esse est percipi: Wer im Netz nicht auffindbar ist, existiert auch nicht. ${ }^{21}$ Wie die Ausführungen zu den Nutzermärkten deutlich gemacht haben, steht die Unentgeltlichkeit der Aufnahme in den Index der Annahme eines eigenen

18 Ibid., Nr. 56. So auch Europäische Kommission, 18.2.2010, COMP/M.5727 - Microsoft/Yahoo! Search Business, Rn. 75. S. die eingehende Diskussion bei Babey, Kartellrechtliche Anforderungen an Suchmaschinen, Diss. 2010, S. 56 ff.

19 Für einen eigenen Markt suchbezogener Werbung beispielsweise Babey (oben Fn. 18),

S. 58; Körber (oben Fn. 15), WRP 2012, 761 (763); Ott, Ich will hier rein! Suchmaschinen und Kartellrecht, MMR 2006, 195 (198); Paal, Netzund Suchmaschinenneutralität im Wettbewerbsrecht, AfP 2011, 521 (526); Zingales, Product Market Definition in Online Search and Advertising, 9 The Competition Law Review 29, 43 f. (2013).

20 So auch Europäische Kommission, 3.10.2014, COMP/M.7217 - Facebook/WhatsApp, Nr. 79; die Untersuchung lege aber in hohem Grad eine Unterteilung der Online-Werbemärkte in suchgebundene und nicht-suchgebundene Werbung nahe, ibid., Nr. 76.

21 Heinemann (oben Fn. 5), S. 14. relevanten Marktes nicht entgegen. Entscheidend für die Annahme eines eigenen Marktes ist die Tatsache, dass es ein Angebot von und eine Nachfrage nach Indexierung gibt. ${ }^{22}$

\section{f) Räumliche Marktabgrenzung}

\section{aa) Nutzermärkte}

16 Google hat sein Suchangebot stark differenziert, indem für die meisten Länder eine eigene Suchfunktion zur Verfügung steht, die durch den Zusatz der länderspezifischen Top Level Domain (z.B. google.ch oder google.de) gekennzeichnet ist. ${ }^{23}$ Die Suchergebnisse für denselben Suchbegriff variieren zwischen den Landesversionen der Suchmaschine, so dass keine beliebige Austauschbarkeit besteht. ${ }^{24}$ Die Bedeutung der Landesversionen ist so gross, dass der Nutzer bei Eingabe von «google.com» offenbar auf die Suchmaschinenversion des eigenen Landes umgeleitet wird. Auch unterscheidet sich die Beliebtheit von Suchmaschinen je nach Land teilweise beträchtlich voneinander. Dies spricht für eine nationale Marktabgrenzung von Nutzermärkten. ${ }^{25}$ Wenn die Annahme korrekt ist, dass Nutzer im Fall von Qualitätsunterschieden zu anderen Landesversionen derselben Sprache wechseln,

$2 2 \longdiv { \text { Für die Annahme eines Marktes für die Aufnahme } }$ in den Suchmaschinenindex auch Höppner (oben Fn. 9), WRP 2012, 625 (626); Paal, Immaterialgüter, Internetmonopole und Kartellrecht, GRUR 2013, 873(876); Weber/Volz, Online Marketing und Wettbewerbsrecht, 2011, Rn. 388.

23 S. die Auflistung unter <http://en.wikipedia.org/wiki/List_of_Google_dom ains $>$.

24 Man gebe z.B. das Wort «Bundesrat» in google.ch und google.de ein.

25 In diesem Sinn Hopf, Der Missbrauch einer marktbeherrschenden Stellung von Internetsuchmaschinen, dargestellt am Beispiel von Google, 2014, S. 73. 
ist aber auch eine geographische Marktabgrenzung nach Sprachräumen plausibel.

\section{bb) Werbemärkte}

Im Bereich der Werbung hat die Europäische Kommission die Märkte in Bezug auf die Bereitstellung von OnlineWerbeflächen national bzw. nach Sprachgrenzen abgegrenzt und hierfür auf nationale Vorlieben, die Sprache und kulturelle Besonderheiten verwiesen. ${ }^{26}$

\section{cc) Aufnahme in den Index}

18 Was den Markt für die Aufnahme in den Index betrifft, so besteht die Besonderheit darin, dass Suchmaschinenindizes weltweit einheitlich erstellt werden. Dies spricht für die Annahme eines Weltmarktes. ${ }^{27}$

\section{g) Fazit}

Suchmaschinen konstituieren dreiseitige Märkte, nämlich Nutzermärkte, Werbemärkte und einen Markt für die Aufnahme in den Index. Zwischen diesen drei Seiten bestehen starke Externalitäten, so dass eine typische mehrseitige Plattform im Sinn der neueren Industrieökonomik vorliegt.

\section{Marktbeherrschende Stellung}

20 Häufig werden Suchmaschinen als gatekeeper bezeichnet, also als Wächter, die das Tor zum Netz bewachen. ${ }^{28}$ Dieses Bild suggeriert eine Unausweichlichkeit, insbesondere der führenden Suchmaschine, die an andere wesentliche Ein-

26 Europäische Kommission, 11.3.2008, COMP/M.4731 - Google/DoubleClick, Nr. 83 f.

27 Babey (oben Fn. 18), S. 70 f.; Hopf (oben Fn. 25), S. 83.

28 S. z.B. Schulz/Held/Laudien, Suchmaschinen als Gatekeeper in der öffentlichen Kommunikation, 2005. richtungen, z.B. unumgängliche Infrastrukturen denken lässt. ${ }^{29}$ Wie bereits die Existenz der Microsoft-Suchmaschine und anderer Suchmaschinen zeigt, ist es aber durchaus möglich, andere Suchmaschinen zu entwickeln. Es ist deshalb eher angemessen, die GoogleSuchmaschine als gateway in das Internet und nicht als gatekeeper zu bezeichnen.30 Die Prüfung einer marktbeherrschenden Stellung geht auch bei mehrseitigen Plattformen von den relevanten Einzelmärkten aus. ${ }^{31}$

\section{a) Nutzermärkte}

21 Die Zahlen über die Nutzung von Suchmaschinen variieren je nach Quelle. In den meisten EU-Mitgliedstaaten (und auch in der Schweiz) liegt der Anteil von Google bei über 90 Prozent aller Suchanfragen. Hohe Marktanteile lösen zwar Vermutungswirkungen aus. ${ }^{32}$ Gerade auf dem Gebiet der Informationstechnologie mit ihren besonderen ökonomischen Eigenschaften ist aber eine Gesamtanalyse erforderlich. So hat das Gericht der Europäischen Union in der Entscheidung zum Zusammenschluss von Microsoft und Skype ausgeführt, dass selbst Marktanteile von 80 bis 90 Prozent nicht notwendigerweise $\mathrm{zu}$ einer erheblichen Behinderung wirksamen Wettbewerbs

$29 \overline{\text { Höppner (oben Fn. 9), WRP 2012, } 625 \text { (637), stellt }}$ Gemeinsamkeiten der Google-Suchmaschine mit klassischen wesentlichen Einrichtungen fest.

30 So Körber (oben Fn. 15), WRP 2012, 761 (765 f.) mwN.

31 Dewenter/Rösch (oben Fn. 11),

S. 119; Monopolkommission (oben Fn. 9), Nr. 29.

32 S. EuGH, 3.7.1991, Rs. C-62/86 - AKZO/Kommission, Slg. 1991, I-3359, Rn. 60: Beweis für das Vorliegen einer marktbeherrschenden Stellung bei einem Marktanteil von 50 Prozent; Europäische Kommission, Leitlinien zur Bewertung horizontaler Zusammenschlüsse gemäss der Ratsverordnung über die Kontrolle von Unternehmenszusammenschlüssen, AB1. 2004, C 31/5, Nr. 17. 
(Significant Impediment to Effective Competition, SIEC) führen, wenn der Markt stark expansiv ist und die Einführung von (pekuniären) Preisen die Nutzer zur Abwanderung zu einem anderen Dienst veranlasst. 33

Ausserdem bestehen im Zusammenhang mit Suchmaschinen keine direkten Netzwerkeffekte: Der Nutzen des Suchenden steigt nicht unmittelbar mit der Anzahl anderer Nutzer derselben Suchmaschine.34 Dies unterscheidet Suchmaschinen von sozialen Netzwerken wie Facebook, bei denen der Nutzen mit jedem weiteren Teilnehmer steigt.35 Im Unterschied zu sozialen Netzwerken besteht bei Suchmaschinen deshalb auch kein lock in: Ohne Mühe und Kosten kann sich der Nutzer einer anderen Suchmaschine zuwenden, ganz im Sinn des von Google angeführten Satzes «Competition is just one click away». Multi-homing ist also ohne weiteres möglich. ${ }^{36}$ Auch die Möglichkeit zur Personalisierung von Suchvorgängen hat hieran nichts geän-

33 EuG, 11.12.2013, Rs. T-79/12 - Cisco Systems und Messagenet/Kommission, ECLI:EU:T:2013:635, Rn. 51 ff., 65 ff. S. die Diskussion bei Graef, Sneak Preview of the Future Application of European Competition Law on the Internet?: Cisco and Messagenet, 51 CMLR 1263 ff. (2014).

34 Haucap/Heimeshoff, Google, Facebook, Amazon, eBay: Is the Internet driving competition or market monopolization?, 11 International Economics and Economic Policy 49, 54 (2014). Zwar erlauben höhere Nutzerzahlen dem Suchmaschinenbetreiber aufgrund der grösseren Datenmenge die Verbesserung des Algorithmus; dieser Effekt sollte aber als Grössenvorteil klassifiziert werden.

35 S. Gebicka/Heinemann, Social Media \& Competition Law, 37 World Competition 149, 160 f. (2014).

36 S. Crane (oben Fn. 7), 8 Journal of Competition Law \& Economics 459, 464 (2012), der Marktbeherrschung von Google aus diesem Grund für unwahrscheinlich hält. In diesem Sinn auch Kersting/Dworschak, ifo Schnelldienst 16/2014, $7 \mathrm{ff}$. dert.

23 Für die Annahme beträchtlicher Verhaltensspielräume sprechen aber neben den bedeutenden Nutzeranteilen in allen Sprachräumen der Europäischen Union bzw. des Europäischen Wirtschaftsraums (sowie der Schweiz) die hohen Marktzutrittsschranken. Im Zusammenhang mit Suchmaschinen sind Grössen- und Verbundvorteile (economies of scale and scope) von besonderer Bedeutung. Mengenausweitungen erlauben die Absenkung der Stückkosten, also der Kosten pro Suchanfrage. Ausserdem steigt die Qualität der Suchmaschine mit der Zahl der Suchanfragen, da deren Auswertung Verbesserungen des Algorithmus erlaubt.37 Die Grössenvorteile erschweren den Marktzutritt anderer Unternehmen. ${ }^{8}$ Verbundvorteile wirken in dieselbe Richtung: Neue Funktionen, welche die klassische Internetsuche ergänzen, können aufgrund von Synergien günstiger angeboten werden und machen die betreffende Suchmaschine noch attraktiver. So bietet Google neben der Suchfunktion Dutzende weiterer Dienstleistungen an. Andere Suchmaschinen oder

$37 \overline{\text { Skeptisch Manne/Wright }}$, Google and the Limits of Antitrust: The Case Against the Case Against Google, 34 Harvard Journal of Law \& Public Policy 171, 211 f. (2011): Die für einen guten Algorithmus nötige Mindestnutzerzahl sei schnell erreicht. DifferenzierterHaucap/Kehder, Suchmaschinen zwischen Wettbewerb und Monopol: Der Fall Google, $2013<w w w . d i c e . h h u . d e / f o r s c h u n g-d i c e / d i c e-$ ordnungspolitische-perspektiven.html>, S. 6 f., 13 ff.: Der Grenznutzen zusätzlicher Nutzerdaten sinkt, so dass ab einer bestimmten Informationsmenge die Grenzkosten den Grenznutzen erreichen und keine weiteren Grössenvorteile mehr entstehen.

38 Vgl. auch Monopolkommission (oben Fn. 9), Nr. 18: Auch wenn Kostensubadditivitäten ein natürliches Monopol begründen können, sind auf den Märkten der Informationstechnologie Innovationen bedeutender als Kostenvorteile. 
Newcomer können mit dieser Vielfalt nicht Schritt halten.39 Ausserdem bestehen indirekte Netzwerkeffekte, da die Suchmaschine umso wertvoller für Inhalteanbieter und die werbende Wirtschaft ist, desto mehr Nutzer von ihr Gebrauch machen. 40

24 Schliesslich sind verhaltensbasierte Faktoren zugunsten einer marktbeherrschenden Stellung von Google anzuführen. Offenbar besteht auch auf den Suchmaschinenmärkten eine starke Pfadabhängigkeit: Es ist schwierig für Newcomer, sich gegenüber dem etablierten Suchmaschinenbetreiber zu behaupten, obwohl keine direkten Netzwerkeffekte bestehen. Selbst die im Jahr 2009 lancierte Suchmaschine Bing verharrt in Europa weiterhin bei Nutzeranteilen im tiefen einstelligen Prozentbereich, obwohl die Markzutrittsschranke des hohen Investitionsbedarfs für den Betreiber Microsoft überwindbar ist. Offenbar spielt der Bekanntheitsgrad bei Suchmaschinen eine besonders wichtige Rolle. ${ }^{41}$ Die Nutzer googeln ${ }^{2}$ meistens, aber bingen43 nur selten. Die Klick-Entscheidung ist habitualisiert.44 Die meisten Nutzer haben in ihrem Inneren die führende Suchmaschine als Default eingestellt. Offenbar sind die Wettbewerber doch weiter als einen Klick entfernt.

\footnotetext{
$39 \mathrm{Zu}$ Portfolio- und Konglomerateffekten s. Monopolkommission (oben Fn. 9), Nr. 24 f.

40 Dewenter/Rösch (oben Fn. 11), S. 242; Haucap/Kehder (oben Fn. 37), S. 4; ablehnend Kühling/Gauß, Expansionslust von Google als Herausforderung für das Kartellrecht, MMR 2007, 751 (752).

41 Weber, Rechtsfragen rund um Suchmaschinen, 2003, S. 113.

42 Das Verb findet sich seit 2004 im Duden.

43 Das Verb findet sich nicht im Duden.

44 Heinemann (oben Fn. 5), S. 22; s. auch Dewenter/Rösch (oben Fn. 11), S. 32.
}

\section{b) Werbemärkte}

25 Nimmt man separate relevante Märkte für Suchmaschinenwerbung an, hat Google nach den Feststellungen der Europäischen Kommission in der Google/DoubleClick-

Fusionsentscheidung aus dem Jahr 2008 Marktanteile im EWR zwischen 40 und 80 Prozent. Grenzt man weiter ab, nämlich allgemein nach Onlinewerbung, liegt der Marktanteil von Google im EWR zwischen 20 und 40 Prozent.45 Da die räumlich relevanten Werbemärkte national bzw. nach Sprachgrenzen abzugrenzen sind, sind allerdings detailliertere Feststellungen erforderlich. Die von der Europäischen Kommission festgestellten Marktanteile nach Ländern liegen für die Suchmaschinenwerbung zwischen 15 und 95 Prozent, für Onlinewerbung insgesamt zwischen «tiefer als zehn Prozent» und 70 Prozent. 46

26 Die Frage der Marktbeherrschung ist also differenziert nach Land bzw. Sprachraum zu beurteilen. Nimmt man Märkte für Suchmaschinenwerbung an, ist im deutschen Sprachraum angesichts hoher Marktanteile von einer marktbeherrschenden Stellung auszugehen. 47

\section{c) Aufnahme in den Index}

27 Weltweit ist der Anteil von Google an den Suchanfragen zwar niedriger als in der Europäischen Union bzw. im Europäi-

45 Europäische Kommission, 11.3.2008, COMP/M.4731 - Google/DoubleClick, Rn. 98, 101.

46 Ibid., Rn. 99, 102.

47 So für Deutschland Hopf (oben Fn. 25), S. 108; für die Schweiz Weber/Volz, Online-Werbemarkt und Kartellrecht - Innovation vs. Marktmacht, sic! 2010, 777 (781 f.). Eine marktbeherrschende Stellung auf dem Markt für suchbasierte Werbung bejaht auch Körber (oben Fn. 15), WRP 2012, 761 (763 f.) 
schen Wirtschaftsraum, aber immer noch sehr hoch. Ausserdem ist der Abstand zu den Wettbewerbern auch auf Weltebene enorm. Dies spricht - zusammen mit den anderen für die Nutzermärkte diskutierten Umständen - für die Annahme einer beherrschenden Stellung auf dem Markt für die Aufnahme in den Index. ${ }^{48}$

Die Feststellung einer marktbeherrschenden Stellung von Google auf dem Markt für die Aufnahme in den Index ist allerdings mit der Differenzierung zwischen search dominance und referral dominance angegriffen worden. Selbst wenn Google eine beherrschende Stellung auf den Suchmärkten hätte (was von den Verfechtern dieses Arguments bestritten oder zumindest für unwahrscheinlich gehalten wird), folge hieraus nicht, dass das Unternehmen auch für das Auffinden von Seiten im Netz dominant sei. Die Benutzung einer Suchmaschine sei nur eine Möglichkeit unter mehreren. Die Nutzer könnten Webadressen auch selbst eingeben, bzw. gelangten über Links auf anderen Websites oder in e-mails, über soziale Medien oder Werbung zum Ziel. Der Traffic auf Websites werde nur zu einem relativ kleinen Prozentsatz durch Suchmaschinen generiert. 49

Dem Argument ist zuzugestehen, dass auch im Internet viele Wege nach Rom führen. Allerdings ist dieser Umstand nur relevant, wenn der Nutzer bereits weiss, dass er nach Rom möchte, d.h. welche Seite er ansteuern will. Er kann dann beispielsweise ins Eingabefeld seines Browsers die Webadresse einfügen

48 Ebenso Hopf (oben Fn. 25), S. $104 \mathrm{ff}$.

49 Crane (oben Fn. 7), 8 Journal of Competition Law \& Economics 459, 464 ff. (2012). oder auf ein bereits abgelegtes Lesezeichen zurückgreifen. In den meisten Fällen hilft dieser Weg allerdings nicht weiter: Der Nutzer weiss nämlich nicht, wo er die gesuchte Information findet und schaltet eben $\mathrm{zu}$ diesem Zweck die Suchmaschine ein. Um den Wettbewerbsdruck zu ermitteln, unter dem eine Suchmaschine steht, ist also auf die typische Suchsituation abzustellen. In dieser ist direktes Ansteuern genauso wenig von Bedeutung wie der Rückgriff auf Links, Werbung oder soziale Medien. Das Konzept der referral dominance wird diesem Umstand nicht gerecht, da es die Perspektive des Findens und nicht diejenige des Suchens einnimmt.

\section{d) Fazit}

3o Google ist im Besitz einer marktbeherrschenden Stellung auf den Nutzer- und Werbemärkten sowie auf dem Markt für die Aufnahme in den Index. Die marktbeherrschende Stellung ergibt sich aus hohen Marktanteilen, die über viele Jahre auf hohem Niveau geblieben sind, im Zusammenwirken mit Grössen- und Verbundvorteilen sowie indirekten Netzwerkeffekten und eingeschliffenen Nutzergewohnheiten. Die marktbeherrschenden Stellungen bestehen auf dem «Binnenmarkt oder auf einem wesentlichen Teil desselben» i.S. von Art. 102 Abs. 1 AEUV, da die relevanten Märkte zumindest national abzugrenzen sind, und die Territorien der grösseren Mitgliedstaaten als wesentliche Teile des Binnenmarktes gelten. $5^{0}$ Auch marktbeherrschende Stellungen i.S. von Art. 7 Abs. 1 KG sind zu bejahen. 5o Zu Letzterem s. Fuchs/Möschel, in Immenga/Mestmäcker (Hrsg.), Wettbewerbsrecht, Band 1. EU/Teil 1, 5. Aufl. 2012, Art. 102 AEUV Rn. 67. 


\section{Missbrauch}

31 Die Europäische Kommission hat ihren Missbrauchsvorwurf auf vier Verhaltensweisen gestützt. ${ }^{51}$ Google bevorzuge die Anzeige der eigenen spezialisierten Suchdienste gegenüber konkurrierenden spezialisierten Suchdiensten. Originalinhalte der Konkurrenten würden von Googles spezialisierten Suchdiensten unautorisiert übernommen. Die Betreiber von Webseiten würden gezwungen, den gesamten oder einen grossen Teil ihres Bedarfs an Suchmaschinenwerbung über Google zu decken. Und schliesslich werde die Übertragbarkeit von SuchmaschinenWerbekampagnen von Google auf konkurrierende Plattformen sowie das plattformübergreifende Management von Suchmaschinen-Werbekampagnen vertraglich beschränkt. Die Vorwürfe zielen in verschiedene Richtungen und bedürfen deshalb separater Betrachtung.

\section{Privilegierung eigener Suchdienste}

32 Die entscheidende Frage lautet: Darf ein dominanter Suchmaschinenbetreiber seine eigenen Dienste gegenüber denjenigen der Konkurrenz bevorzugen?52 Das Kriterium des Leistungswettbewerbs spricht dafür, dass Wettbewerber die Möglichkeit haben sollen, durch bessere, innovativere und/oder billigere Produkte

51 S. Europäische Kommission, Pressemitteilung IP/13/371 vom 25.4.2013, S. 2. Andere Vorwürfe werden derzeit geprüft, z.B. die Frage, welche Verhaltensanforderungen aus europäischem Kartellrecht für die Ausgestaltung des AndroidBetriebssystems für Mobilfunkgeräte und Tabletcomputer folgen. S. hierzu Körber, Machtmissbrauch durch Android? - Zum Wettbewerb auf den Märkten für mobile Betriebssysteme und Anwendungen, NZKart 2014, 378 ff.; Paal (oben Fn. 22), GRUR 2013, 873 (879 f.).

52 Zur Ökonomik eines solchen «search bias» s. Haucap/Kehder (oben Fn. 37), S. 10 ff.
Erfolg am Markt zu haben. Dieses Ziel wird konterkariert, wenn Marktbeherrschung im Zusammenhang mit horizontaler Suche eingesetzt wird, um konkurrierende Vertikalsuchdienste $\mathrm{zu}$ behindern. Zwar kann das betreffende Verhalten nicht unter den Diskriminierungstatbestand (Art. 102 Abs. 2 lit. c AEUV / Art. 7 Abs. 2 lit. b KG bzw. die Generalklauseln) gefasst werden, da die Diskriminierung zwischen Drittunternehmen und nicht im Verhältnis der eigenen Dienste des Marktbeherrschers zu solchen Dritter erfolgen muss.53 Es liegt aber eine Kopplung vor.54 Die vertikalen Dienste werden im Zusammenhang mit der horizontalen Suche angeboten.55 Ihre Informationen werden den «organischen» Suchergebnissen hinzugefügt, und zwar häufig an prominenter Stelle. Es besteht die Gefahr der Marktverschliessung, da die Nutzer keinen Anlass haben, auf konkurrierende vertikale Dienste zurückzugreifen.

53 Fuchs/Möschel, in Immenga/Mestmäcker (Hrsg.), Wettbewerbsrecht, Band 1.EU/Teil 1, 5. Aufl. 2012, Art. 102 AEUV Rn. 377.

54 S. Edelman, Leveraging Market Power through Tying and Bundling: Does Google Behave AntiCompetitively?, Harvard Business School Working Paper 14-112, 2014; vgl. auch Europäische Kommission, Erläuterungen zu den Prioritäten der Kommission bei der Anwendung von Artikel 82 des EG-Vertrags auf Fälle von Behinderungsmissbrauch durch marktbeherrschende Unternehmen, AB1. 2009 C 45/7, Rn. 47 ff.

55 Der Einwand, horizontale und vertikale Dienste stellten ein einheitliches Produkt dar, entspricht nicht den gegenwärtigen Verhältnissen, s. Hopf (oben Fn. 25), S. 136. Für die Annahme getrennter Produkte reicht es aus, dass Dritte das betreffende Produkt auch separat anbieten,

s. Europäische Kommission, 24.3.2004, COMP/37.792 - Microsoft, Rn. 804. S. näher Bulst, in Langen/Bunte (Hrsg.), Kartellrecht, Kommentar - Band 2: Europäisches Kartellrecht, 12. Aufl. 2014, Art. 102 AEUV Rn. 225. 
33 Google hat Verpflichtungszusagen vorgeschlagen, nach denen das Unternehmen auch die Dienste dreier Konkurrenten anzeigt, wenn es eigene spezialisierte Suchdienste anbietet. Die Auswahl der drei Wettbewerber soll aufgrund eines objektiven Selektionsmechanismus erfolgen. ${ }^{6}$ Bei den eigenen Suchdiensten soll Google als Leistungserbringer ausdrücklich genannt werden. Die spezialisierten Suchdienste sollen von den natürlichen Suchergebnissen graphisch getrennt werden. Ausserdem soll visuelle Vergleichbarkeit mit den Konkurrenzprodukten hergestellt werden. Wenn beispielsweise in den Google-Diensten Bilder gezeigt werden, dann sind auch bei den konkurrierenden Diensten Bilder einzubauen. 57

34 Es ist ungewiss, ob es $\mathrm{zu}$ einem $\mathrm{Ab}$ schluss des Verfahrens auf dem Weg der Verpflichtungszusagen kommen wird. 58 Nach der hier vertretenen Auffassung hätten die soeben geschilderten Massnahmen für eine bessere Sichtbarkeit konkurrierender Vertikalsuchdienste grosse Vorteile.59 Einerseits kommt es nicht $\mathrm{zu}$ einer unangemessenen Einmischung in die Freiheit des Marktbeherrschers zur Gestaltung seiner Produkte, da er weiterhin spezialisierte Suchdienste im Zusammenhang mit der allgemeinen Suchmaschine anbieten kann. Anderer-

${ }_{56} \overline{\text { Soweit es um vertikale Dienste geht, die kommerzi- }}$ ell vermarktet werden, soll die Selektion in Form einer Auktion erfolgen mit Zahlungspflichten nach dem pay per click-Prinzip. Wo nicht monetarisiert wird, sollen die drei selektionierten Wettbewerber auch nicht zahlungspflichtig sein.

57 S. Europäische Kommission, MEMO/14/87 vom 5.2.2014, S. $1 \mathrm{f}$.

58 S.o. I.

59 Grundsätzlich positiv zu den vorgeschlagenen Verpflichtungszusagen auch Haucap/Kehder, ifo Schnelldienst 16/2014, 3 (5). seits wird dem Grundsatz des Leistungswettbewerbs Genüge getan. Dem Nutzer wird die tatsächliche Möglichkeit eingeräumt, von der Existenz konkurrierender Vertikaldienste Kenntnis zu nehmen und auf dieser Grundlage eine informierte Entscheidung zu treffen. Damit erhöht sich die Wahrscheinlichkeit, dass nicht die Ubiquität, nämlich der auf der marktbeherrschenden Stellung beruhende Vertriebsvorteil, sondern die Qualität über den Erfolg des Produkts entscheidet.

\section{2. Übernahme der Inhalte von Kon- kurrenten}

35 Der zweite Vorwurf betrifft die unautorisierte Verwendung von Originalinhalten Dritter in den spezialisierten Suchdiensten von Google, z.B. in Bezug auf journalistische Produkte oder auf nutzergenerierte Inhalte (user-generated content), wie z.B. Restaurant- oder Hotelbewertungen. Aufgrund des von den Suchmaschinen akzeptierten Robots Exclusion Protocol (REP) können die Inhalteanbieter unter Verwendung der Datei robots.txt ihre Website für Suchmaschinen ganz oder teilweise sperren. $\mathrm{Zu}$ einem entsprechenden Ergebnis führt der Einsatz von Meta-Elementen im HTMLQuelltext von Websites wie z.B. «noindex» oder «nofollow».6o Die Suchmaschinen bieten auch an, Seiten auf Antrag direkt an den Suchmaschinenbetreiber aus den Suchergebnissen zu entfernen.

36 Betroffene beschwerten sich, dass die Nutzung technischer Schutzvorkehrungen häufig dazu führe, dass die eigenen Inhalte nicht lediglich aus den Googleeigenen Vertikaldiensten entfernt wür-

6o S. näher Hürlimann (oben Fn. 3), S. 19 ff., 68 ff. 
den, sondern dass die Seiten gänzlich aus der allgemeinen Suchmaschine, nämlich der organischen Trefferliste verschwänden. Der Einsatz der dominanten Suchmaschine zu dem Zweck, Inhalteanbieter davon abzuhalten, die Übernahme ihrer Inhalte in die vertikalen Suchdienste des Marktbeherrschers durch technische Massnahmen zu verhindern, wäre in der Tat als missbräuchlicher Marktmachttransfer zu werten. ${ }^{61}$ Es ist deshalb konsequent, dass eine der Verpflichtungszusagen darin besteht, Inhalteanbietern die Möglichkeit zum Ausschluss aus Googles Vertikaldiensten einzuräumen, ohne Nachteile bei der allgemeinen Suche (oder dem AdWords-Programm) zu erleiden.

\section{Ausschliesslichkeitsbindungen bei Suchmaschinenwerbung}

37 In der Prioritätenliste zu Art. 102 AEUV hat die Europäische Kommission die Ausschliesslichkeitsbindungen durch Marktbeherrscher an die erste Stelle gesetzt. ${ }^{62}$ Die Vereinbarung von Alleinbezugsbindungen mit den Abnehmern kann Wettbewerbern die Absatzkanäle versperren (customer foreclosure). Die Europäische Kommission schreitet prioritär in den Fällen ein, in denen Alleinbezugsbindungen dazu führen, dass der Markteintritt bzw. die Expansion konkurrierender Unternehmen verhindert wird. ${ }^{63}$

61 Soweit die genannten Inhalte urheberrechtsgeschützt sind, kommt es zu der interessanten Konstellation, dass das Kartellrecht Immaterialgüterrechte schützt. Beim Verhältnis des Kartellrechts zum Immaterialgüterrecht stehen ansonsten immer die Fälle im Zentrum der Aufmerksamkeit, in denen beide Rechtsgebiete miteinander kollidieren, vgl. Heinemann, Immaterialgüterschutz in der Wettbewerbsordnung, 2002, S. $470 \mathrm{f}$.

62 Europäische Kommission, Erläuterungen zu den Prioritäten (oben Fn. 54), Rn. 32 ff.

63 Ibid., Rn. 34.
Das ist insbesondere dann der Fall, wenn der Marktbeherrscher ein nicht zu umgehender Handelspartner ist. ${ }^{64}$ Nach den Feststellungen der Europäischen Kommission hatte Google mit den Betreibern von Websites Vereinbarungen geschlossen, welche diese de facto an Google banden, die Vermittlung von Werbung durch andere Dienstleister nämlich grösstenteils ausschlossen. Angesichts der überragenden Bedeutung der GoogleSuchmaschine liegt die Annahme einer wettbewerbswidrigen Marktverschliessung nahe. Auf Exklusivitätsbindungen ist deshalb zu verzichten.

\section{4. Übertragbarkeit von Werbekam- pagnen}

38 Wettbewerber werden nach den Feststellungen der Europäischen Kommission auch dadurch behindert, dass Google die Möglichkeit zur Übertragung der Werbekampagnen vom AdWords-Programm auf konkurrierende Plattformen für Suchmaschinenwerbung einschränkt. So würden Softwareentwickler daran gehindert, Werkzeuge anzubieten, welche eine Verwaltung von Werbekampagnen über mehrere Plattformen ermöglichen. ${ }^{65}$ Einschränkungen der Datenübertragbarkeit können in der Tat faktisch zum Ausschluss von Wettbewerbern führen. Die von Google vorgeschlagenen Verpflichtungszusagen sehen deshalb zu Recht vor, dass auf Einschränkungen der Portabilität von Werbekampagnen zu verzichten ist. Zu einem entsprechenden Ergebnis ist auch das Kartellverfahren in den USA gelangt. ${ }^{66}$

64 Vgl. ibid., Rn. 36.

65 Europäische Kommission, MEMO/14/87 vom 5.2.2014, S. 2.

66 Federal Trade Commission, Pressemitteilung vom 3.1.2013 (oben Fn. 2). 


\section{Suchneutralität?}

39 Die vorstehend genannten Missbrauchsformen sind das Ergebnis einer Selektion, welche die Europäische Kommission unter einer Vielzahl von Beschwerden vorgenommen hat. Es steht ersichtlich das Bemühen im Vordergrund, möglichst spezifische Verhaltensweisen zu identifizieren, um klare kartellrechtliche Vorgaben zu erreichen. Nicht behandelt wird die «klassische» Frage, ob aus dem Missbrauchsverbot auch ein Objektivitätsgebot für die marktbeherrschende Suchmaschine folgt. Unternehmen (oder allgemein Betreiber von Websites), die ihre Platzierung in den Trefferlisten als zu schlecht empfinden, mahnen seit jeher «Suchneutralität» auf der Grundlage objektiver Kriterien an und zeihen Google des search bias. ${ }^{67}$

40 Die Diskussion dieser Frage hat mehrheitlich zu folgenden Differenzierungen geführt: Aus Art. 102 AEUV / Art. 7 KG folgt ein Anspruch auf Aufnahme von Websites in den Index der Suchmaschine. ${ }^{68}$ Eine Nichtberücksichtigung stellt eine Leistungseinschränkung i.S. von Art. 102 Abs. 2 lit. b AEUV / Art. 7 Abs. 2 lit. e KG und eine unzulässige Diskrimi-

67 S. beispielsweise die Nachweise bei van Loon (oben Fn. 16), S. 16 f., und die Untersuchung von Erhard, Marktmachtverlagerung durch Suchmaschinenbetreiber - Suchmaschinenneutralität im europäischen, deutschen und US-amerikanischen Kartellrecht, 2014.

68 Die Frage, ob die zivilrechtlichen Ansprüche wegen Verletzung europäischen Kartellrechts direkt im europäischen Recht wurzeln oder sich lediglich aus nationalem Recht ergeben, hat Erwägungsgrund 8 der Richtlinie 2014/104/EU (AB1. 2014 L 349/1) zum kartellrechtlichen Schadensersatz («unionsrechtlicher Schadensersatzanspruch») zugunsten der ersten Meinung entschieden. Für den Unterlassungsanspruch sollte nichts anderes gelten. Die unionsrechtlichen Ansprüche sind im nationalen Recht zu konkretisieren (für Deutschland s. § 33 GWB). nierung im Vergleich zu den Seiten dar, die in den Index aufgenommen wurden. ${ }^{69}$ Ein Ausschluss von Seiten aus dem Index ist aus sachlichen Gründen möglich:70 Hierzu zählen beispielsweise das Angebot rechts- oder sittenwidriger Inhalte oder die unbillige Manipulation von Seiten zur künstlichen Verbesserung des Rankings (sog. SuchmaschinenSpamming im Gegensatz zur legitimen Suchmaschinen-Optimierung ${ }^{71}$ ).

41 Mit der reinen Aufnahme in den Index ist den Inhalteanbietern in der Regel nicht geholfen. Sie streben vielmehr nach einer möglichst guten Platzierung in der Trefferliste. Die entscheidende Frage lautet also, ob sich aus dem Missbrauchsverbot Mindestanforderungen an die Ausgestaltung des Suchmaschinenalgorithmus ableiten lassen. Wie bereits aus der Unternehmensgeschichte von Google, insbesondere der Entwicklung des PageRank, aber auch der kontinuierlichen Weiterentwicklung des Suchalgorithmus deutlich wird, ${ }^{72}$ gibt es keine objektiv richtige oder optimale Methode zur Reihung der Suchergebnisse. Es bestehen also erhebliche Zweifel daran, ob die Forderung nach Objektivität überhaupt im Bereich des Möglichen liegt.73 Auch ist festzuhalten, dass die Herstellung von Transparenz in Bezug auf den Suchalgorithmus aufgrund der sich zwangsläufig anschliessenden Suchmaschinenoptimie-

69 Heinemann (oben Fn. 5), S. 23 f.

70 S. Hopf (oben Fn. 25), S. 120 ff.

71 S. die Angaben von Google zur Spam-Bekämpfung unter <www.google.com/intl/de/insidesearch/ howsearchworks/fighting-spam.html $>$. Zu den Schwierigkeiten der Abgrenzung s. Glöggler, Suchmaschinen im Internet, 2003, S. 187 ff.

72 S.o. II.1.a.

73 Weber/Volz (oben Fn. 13), unter III.2.b.cc. Ansätze zu einer Objektivierung des Ranking finden sich bei Hürlimann (oben Fn. 3), S. 78 f. 
rung kontraproduktiv wäre. Ferner geniesst der Schutz des Algorithmus als Betriebsgeheimnis hohen Stellenwert. Aus dem kartellrechtlichen Missbrauchsverbot ist deshalb kein allgemeines Gebot der Suchneutralität abzuleiten. ${ }^{74}$ Ein Anspruch auf eine bestimmte Position im Ranking besteht also nicht. 75

Die Grenze zum Missbrauch wird allerdings bei willkürlicher Aufstellung der Trefferliste überschritten. Eine grundlose Herabstufung im Ranking ist durch das weite Ermessen des Suchmaschinenanbieters nicht mehr gedeckt. ${ }^{76}$ Aus dem Missbrauchsverbot sollten ausserdem Minimalvorgaben für das Verfahren und eine Pflicht zur Wahrung der Verhältnismässigkeit abgeleitet werden.77 Wird beispielsweise eine Website aufgrund von Suchmaschinen-Spamming abgewertet, ist dem Anbieter der Seite die Möglichkeit zu bieten, den Mangel abzustellen und einen Antrag auf erneute Überprüfung zu stellen. ${ }^{78}$ Ausserdem muss ein

74 So für das US-amerikanische Antitrustrecht Crane (oben Fn. 8), 9 I/S: A Journal of Law and Policy for the Information Society 397 (2014). Crane nimmt an, dass auch in Abwesenheit eines Prinzips der Suchneutralität anders begründete Kartellrechtsvorgaben für die Gestaltung von Suchmaschinen möglich bleiben (ibid., 400).

75 Hopf (oben Fn. 25), S. 124, 130, 151.

$76 \operatorname{Hopf}$ (oben Fn. 25), S. 139. Auch diejenigen, die sich gegen ein allgemeines Gebot der Suchneutralität aussprechen, erkennen eine fallbezogene Kontrolle von Extremfällen an, s. z.B. Crane (oben Fn. 7), 8 Journal of Competition Law \& Economics 459, 460 (2012): «Although there may be a limited case for antitrust liability on a factspecific basis for acts of naked exclusion against rival websites, the case for a more general neutrality principle is weak".

77 Zum «Wettbewerbsschutz durch Verfahren» in diesem Zusammenhang s. Heinemann (oben Fn. 5), S. $24 \mathrm{ff}$.

78 Hürlimann (oben Fn. 3), S. 130 ff. Google sieht ein solches Verfahren vor, s. die Angaben unter <https://support.google.com/webmasters/answer/
Verfahren zur Verfügung stehen, das es den Betroffenen erlaubt, sich gegen den Vorwurf der Rechts- oder Sittenwidrigkeit $\mathrm{zu}$ wehren. Die Überprüfung muss der besonderen Marktdynamik angemessen sein, also in angemessener Frist erfolgen.

\section{Der Innovationszusammenhang}

43 Der Innovationsaspekt ist bei Kartellrechtsfällen im Bereich der Informationstechnologie von besonderer Bedeutung, wobei Verkürzungen nicht statthaft sind. Eine Analyse, die ausschliesslich auf die Innovationsanreize des Marktbeherrschers abstellt, wäre eine unzulässige Engführung, auch wenn die Auswirkungen auf den Marktbeherrscher ein wichtiger Bestandteil der Untersuchung sind. $\mathrm{Zu}$ Recht ist vorgebracht worden, dass eine umfassende Pflicht zur Suchneutralität die Anreize des Marktbeherrschers zur Weiterentwicklung seiner Produkte beeinträchtigen würde.79 Auch aus diesem Grund wird hier keine grundsätzliche Neutralitätspflicht angenommen. Auch soll Google in der Gestaltung seiner Dienste im Prinzip frei sein, insbesondere auch seine vertikalen Dienste der allgemeinen Suchmaschine hinzufügen können. ${ }^{80}$ Gleichzeitig sollen aber auch die Innovationsanreize der Wettbewerber erhalten bleiben.

44 Eine Pflicht, neben den eigenen vertikalen Diensten auch die Dienste dreier Konkurrenten anzuzeigen, bringt die Innovationsanreize aller Akteure ins Gleichgewicht: Eine solche Vorgabe hin-

\section{3?hl=de $>$.}

79 Crane (oben Fn. 7), 8 Journal of Competition Law \& Economics 459, 466 f. (2012).

80 S.o. III.1. 
dert den Marktbeherrscher nicht an der Weiterentwicklung seiner Produkte. ${ }^{81}$ Und für die konkurrierenden Anbieter bleiben Anreize zur Hervorbringung innovativer Produkte bestehen, da sie eine Perspektive auf Sichtbarkeit bei der führenden Suchmaschine haben.

Was die anderen Aspekte des Verfahrens betrifft, lassen sich ähnliche Überlegungen anstellen. Die Betreiber von Websites müssen die Möglichkeit haben, ihre Inhalte den vertikalen Suchdiensten von Google vorzuenthalten, ohne Nachteile bei der allgemeinen Suche zu erleiden, da andernfalls ihre Anreize zur Hervorbringung solcher Inhalte beeinträchtigt würden. Der Marktbeherrscher ist demgegenüber nicht daran gehindert, vergleichbare Inhalte selber zu produzieren. Und die Aufhebung faktischer Ausschliesslichkeit in Bezug auf Suchmaschinenwerbung und Werbekampagnen ermöglicht es ebenfalls alternativen Anbietern, neue Leistungen zu entwickeln, ohne den Marktbeherrscher in seinem Innovationspotential zu schmälern.

Schliesslich sei auf folgenden Zusammenhang aufmerksam gemacht: Immaterialgüterschutz wird häufig mit dem Argument begründet, dass der Ausschluss von kurzfristigem Imitations-

81 Anders die Federal Trade Commission im USamerikanischen Kartellverfahren, welche die Einführung von Universal Search und Änderungen des Algorithmus zum Nachteil von Wettbewerbern unter dem Gesichtspunkt der Innovation für gerechtfertigt hält (Federal Trade Commission, Pressemitteilung vom 3.1.2013, oben Fn. 2). Die der Europäischen Kommission vorgeschlagenen Verpflichtungszusagen beschlagen demgegenüber einen Mittelweg. Google hat das Recht zur Weiterentwicklung seiner Produkte; die Wettbewerber behalten aber die Chance, aufgrund besserer Leistungen Erfolg am Markt zu haben. Die Innovationstätigkeit aller Beteiligten wird hierdurch gefördert. wettbewerb den langfristigen Substitutionswettbewerb fördern soll.82 Dieser Grundgedanke lässt sich allgemein für die Auslegung von Art. 102 AEUV / Art. 7 KG in dynamischer Hinsicht fruchtbar machen. Danach sind zum Schutz der Innovationsanreize hohe Voraussetzungen an kartellrechtliches Eingreifen zu stellen. Die Eingriffsschwelle ist aber dann erreicht, wenn die Geschäftsstrategie des Marktbeherrschers dazu führt, dass Substitutionswettbewerb im Keim erstickt wird.

47 Im Bereich der horizontalen Suche hat selbst die durch ein so wichtiges Unternehmen des IT-Sektors wie Microsoft lancierte Suchmaschine Bing nur geringen Nutzerzuspruch gefunden. Dagegen ist auf dem Gebiet der vertikalen Suchmaschinen grosse Dynamik zu verzeichnen. Wenn das Geschäftsmodell des Marktbeherrschers gerade darauf zielt, durch Bevorzugung der eigenen Vertikaldienste die mit diesen konkurrierenden Dienste anderer Anbieter klein zu halten, ist die langfristige Perspektive des Substitutionswettbewerbs gefährdet. In einer solchen Konstellation ist nicht die Anwendung, sondern die Nichtanwendung von Art. 102 AEUV / Art. 7 KG eine Gefahr für die Innovationsanreize. Diese Aussage bezieht sich nicht nur auf die Innovationsanreize Dritter, sondern auch auf die des Marktbeherrschers selber: Können sich die eigenen Dienste nicht auf die Privilegierung durch die beherrschende Suchmaschine verlassen, sondern müssen sie mit Drittanbietern nach dem Leistungskriterium konkurrieren, ist der Verbesserungsdruck noch grösser.

82 S. beispielsweise C.C. von Weizsäcker, Rechte und Verhältnisse in der modernen Wirtschaftslehre, 34 Kyklos 345 (1981). 


\section{Ausblick}

48 Die Märkte der Informationstechnologie zeichnen sich durch ein besonders hohes Innovationstempo aus. Dem Aufstieg von Unternehmen und Produkten kann schnell der Abstieg folgen. Hieraus pauschal eine im Verhältnis zu anderen Märkten eingeschränkte Anwendung des Kartellrechts abzuleiten, wäre aber eine in mehrfacher Hinsicht verkürzte Schlussfolgerung. Erstens gibt es auch im Bereich der Informationstechnologie langlebigen, nämlich länger als zehn Jahre währenden Erfolg. PCBetriebssysteme, nämlich Windows, und eben auch die erfolgreichste horizontale Suchmaschine Google sind Beispiele hierfür. Zweitens verbieten Art. 102 AEUV / Art. 7 KG nicht schon Marktbeherrschung, sondern nur deren Missbrauch. Wie ist es zu beurteilen, wenn ein Marktbeherrscher Geschäftsstrategien anwendet, die gerade der künstlichen Verlängerung des Innovationszyklus dienen? Und drittens ist es im Moment der Kartellrechtsanwendung kaum möglich, die Dauer unternehmerischen Erfolgs zu prognostizieren.

49 Es ist nicht die Aufgabe des Kartellrechts, Besitzstände gegen Strukturwandel oder aktuelle Verdienstmöglichkeiten gegen neue Geschäftsmodelle zu schützen. Wohl aber sind die Marktzutrittsschranken möglichst niedrig zu halten, um auch den Marktbeherrscher der Substitutionskonkurrenz auszusetzen. Eine der wichtigsten Aufgaben des Kartellrechts besteht deshalb darin, die Bestreitbarkeit von Märkten gegen unzulässige Wettbewerbsbeschränkungen $\mathrm{zu}$ verteidigen und hierdurch der Erosion von Markt- macht ihren Lauf zu lassen. ${ }^{83}$ Weder die Netzwerkeffekte noch Grössen- und Verbundvorteile sind das kartellrechtliche Problem, sondern die Ausnutzung unkontrollierter Verhaltensspielräume unter Missachtung des Leistungskriteriums. Marktbeherrschende Unternehmen sollten sich die Gunst ihrer Kunden durch bessere, billigere und innovativere Produkte sichern und nicht durch leistungsferne Behinderungspraktiken. Bei der Konkretisierung dieser Vorgaben ist das Innovationspotential aller Marktakteure zu berücksichtigen. Das Kartellrecht darf Innovationen nicht behindern, sondern soll sie fördern. Es bedarf grosser Anstrengungen, um dieses Ziel zu erreichen.
83 Heinemann, The Contestability of IP-Protected Markets, in Drexl (Hrsg.), Research Handbook on Intellectual Property and Competition Law, 2008, S. $54 \mathrm{ff}$. 